\title{
THE OBSERVED CHARACTERISTICS \\ OF THE LOCAL MAGNETIC FIELD
}

\author{
J. B. WHITEOAK \\ Division of Radiophysics, CSIRO, Sydney, Australia
}

\begin{abstract}
The main investigations of the local magnetic field are reviewed and are found to contain some conflict in interpretation. At radio wavelengths, studies have been made using both the Faraday rotation of the polarized radiation from extragalactic sources and pulsars, and the polarization of the galactic background radiation. With the former type of observation, although more data are available for extragalactic sources, any interpretation may be complicated by the influence of distant field structure. The results are consistent with a large-scale field parallel to the galactic plane, with a field strength of about $2 \mu \mathrm{G}$, and which is directed towards $l=90^{\circ}$. This field contains irregularities in direction and strength on a scale of about $100-200 \mathrm{pc}$. The polarization of galactic background radiation may yield the most detailed information about the local field structure - the results to date show loops of magnetic fields extending along the radio spurs.

The interpretation in terms of small-scale irregularities embedded in a large-scale field parallel to the galactic plane differs from that proposed to explain the optical polarization of starlight, in which a helical field configuration near the Sun was preferred to a more disordered pattern.
\end{abstract}

\section{Introduction}

A magnetic field is an important constituent of the Galaxy. On theoretical grounds it is believed by some (e.g. Piddington, 1972) to play a major role in the formation and evolution of galactic spiral structure. The existence of the field is manifested in many ways: in the Faraday rotation of extragalactic polarized radiation, the presence of galactic synchrotron radiation, the Zeeman splitting of spectral lines, and the optical polarization of starlight. However, the investigation of it is similar to that of spiral structure in its perversity, in that the more data are amassed, the more uncertain the solution becomes. Despite numerous studies, no satisfactory interpretation has been obtained for the structure of the local magnetic field. In this review I will attempt to describe the most widely accepted features by an appraisal of the various results, some of which appear to be in conflict. (The variation in point of view is demonstrated well in the differing conclusions of the reviews by Verschuur (1970) and Verschuur (1972).)

\section{Results at Radio Wavelengths}

\section{(a) THE DISTRIBUTION OF FARADAY ROTATION}

\section{(i) Polarization of Extragalactic Radio Sources}

One of the main methods used to investigate the local magnetic field is based on the Faraday rotation of the polarized radiation from extragalactic radio sources. The Faraday rotation is proportional to the product of electron density $n_{\mathrm{e}}$ and the line-ofsight component of magnetic field $B_{L}$, with the product integrated along the line of sight. Therefore, provided the electron density and magnetic field strength are reason- 
ably constarit, the distribution of rotation with galactic coordinates should provide information about the structure of the magnetic field. The method appeared promising in the initial observations of Gardner and Whiteoak (1963), where it was shown that the average rotation decreased as expected with increasing galactic latitude. However, later investigations of the variation with latitude and longitude were made by Morris and Berge (1964), Gardner and Davies (1966), Berge and Seielstad (1967) Gardner et al. (1969), Mitton (1972) and Wright (1973), but no simple field configuration was derived. Aside from the possibility that the field possesses complex structure, there are several factors that may contribute to this failure. The rotation intrinsic to the sources is assumed to be small, and although this is generally true, since few sources at high latitudes have large rotation measures, some exceptions are known, e.g., Cyg A (Hollinger et al., 1964; Mitton, 1973). The rotation measure is derived on the assumption that the direction of polarization is proportional to wavelength squared, and this may not hold for objects with several differently polarized components with different spectra (such as quasars). The greatest limitation is probably that the line of sight for each source passes through the entire galaxy, and so the rotation may be affected not only by the local magnetic field but also by distant fields. This may even apply at high latitudes in the directions of the high-velocity clouds of neutral hydrogen which may indicate the presence of outer spiral arms (Davies, 1972; Verschuur, 1973a, b).

Figure 1 shows the distribution with galactic latitude of the rotation measures of about 530 sources observed at Parkes. Many of the values are based on unpublished observations. As found in 1963, the concentration of high rotation measures towards the equator is greater than expected from the distribution of electrons alone, but can be explained in terms of a large-scale magnetic field in the plane of the Galaxy. Figure 2 shows the distribution of rotation measures with respect to galactic coordinates. The broken lines show the loops of radio emission as depicted schematically by Haslam et al. (1971). The point-to-point variation in rotation measure is generally large, and Lerche (1970) and others have argued that there are large fluctuations in both magnetic field and electron density that dominate any large-scale field configuration that might exist. However, the distribution shows patterns in the sign of the rotation measures that persist over many square degrees, suggesting a large-scale order in the magnetic field. At present, the overall interpretation of the results that is most widely accepted is probably that advanced by Gardner et al. (1969). They argued that the data at negative galactic latitudes are consistent with a large-scale field which is parallel to the galactic plane and is directed towards $l=80^{\circ}$. At northern latitudes, the rotation measures between $l=300^{\circ}$ and $l=30^{\circ}$ are opposite in sign to that expected for this field alone (as was first pointed out by Morris and Berge (1964)), and Gardner et al. (1969) suggested that the reversal could be due to a looped field extending north of the galactic plane and possibly associated with Loop I (which contains the North Polar Spur). At $l=180^{\circ}, b=-30^{\circ}$, and $l=15^{\circ}, b=+10^{\circ}$, the rotation measures are too large in amplitude for directions nearly orthogonal to the field, and Gardner $e t$ al. (1967) concluded that the anomalies were caused by loops of magnetic field ássociated with spurs of gas taking part in the general expansion of Gould's Belt. The more 


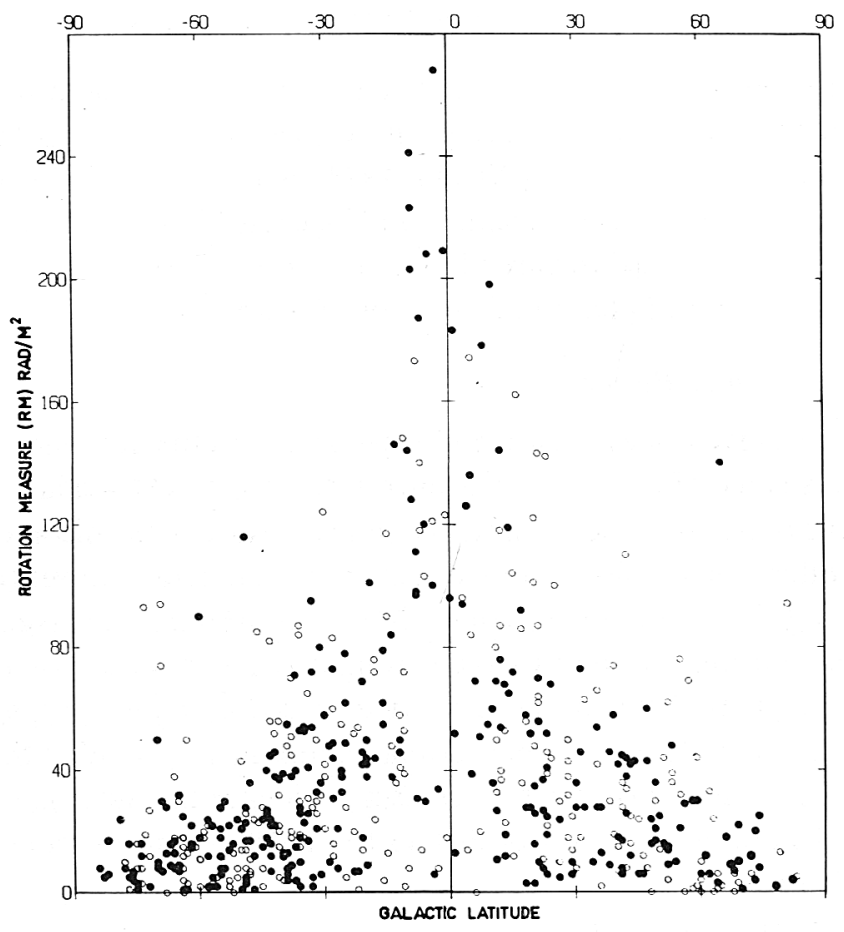

Fig. 1. The distribution with galactic latitude of the rotation measures for sources observed at Parkes. The open circles represent negative values, the closed circles show positive values. Positive values correspond to line-of-sight magnetic fields directed toward the observer.

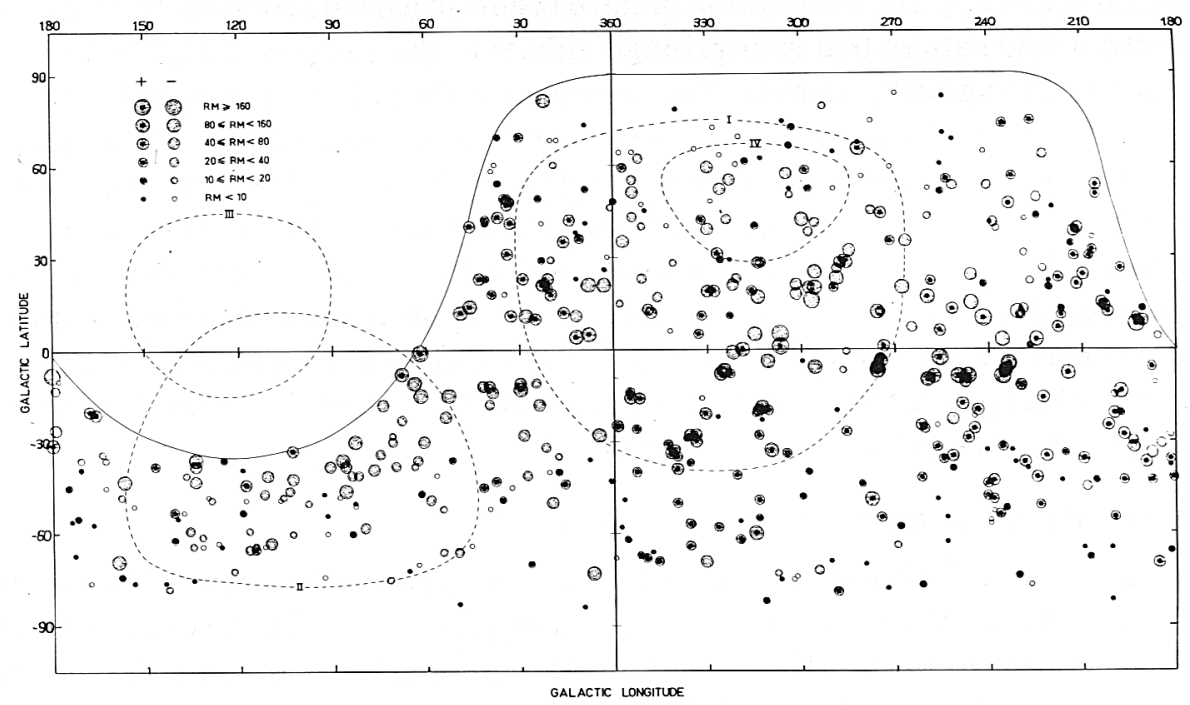

Fig. 2. The distribution with galactic coordinates of the rotation measures for sources observed at Parkes. Positive rotation measures correspond to line-of-sight magnetic fields directed toward the observer. The continuous line shows the limits of observation of the $64-\mathrm{m}$ telescope. The broken lines show the loops of radio emission according to Haslam et al. (1971). 
extensive data shown in Figure 2 suggest that such loops or field irregularities are more common than indicated by the results presented in 1969. In the most recent investigation of Faraday rotation (Wright, 1973), which included for the first time a substantial sample of sources at declinations inaccessible to Parkes, the large-scale field was found to be directed towards $l=94^{\circ} \pm 3^{\circ}, b=-8^{\circ} \pm 8^{\circ}$, and has a strength of about $2 \mu \mathrm{G}$ for an assumed electron density of $0.06 \mathrm{~cm}^{-3}$.

\section{(ii) Faraday Rotation of Pulsars}

The use of pulsars, many of which are highly polarized, in the investigation of Faraday rotation reduces some of the difficulties encountered with extragalactic sources. The dispersion of the pulses provides an approximate measure of the distance of the pulsar and therefore offers the possibility of studying the local field without the influence of more distant magnetic structure. Furthermore, by combining the dispersion measure $(D M)$ with the rotation measure $(R M)$, a weighted mean value for the lie-of-sight component of magnetic field can be directly derived: $\bar{B}_{L}=\int B_{L} n_{\mathrm{e}} d_{l} / \int n_{\mathrm{e}} d_{l} \propto R M / D M$. This value is similar to the mean longitudinal component, provided that the fluctuations in $n_{\mathrm{e}}$ and $B_{L}$ are not correlated. There is no evidence that any significant fraction of the observed Faraday rotation occurs in the pulsar or in its immediate vicinity (Manchester, 1972).

Unfortunately, the pulsars for which both the dispersion measure and rotation measure have been determined are small in number and concentrated near the galactic equator. Therefore only the large-scale features of the magnetic field can be traced. Manchester (1973) has carried out the most comprehensive study using a total of 38 pulsars (Table I). The distribution of these results in galactic coordinates (Figure 3) indicates a field pattern that is surprisingly similar to that deduced using the Faraday rotation of extragalactic sources. The pulsars near the galactic plane, and with calculated distances of less than $2 \mathrm{kpc}$, delineate a large-scale field of $2.2 \mu \mathrm{G}$ that is aligned parallel to the plane and directed towards $l=94^{\circ} \pm 11^{\circ}$. The sense and strength of the field in the direction of the North Polar Spur show anomalies similar to those encountered with the extragalactic sources. The field is more uniform than deduced from extragalactic sources, so the influences of distant structure and intrinsic Faraday rotation may be responsible for many of the small-scale irregularities evident in Figure 2. Clearly, a larger sample of pulsars is needed before the results for the two groups of objects can be compared in detail.

\section{(iii) Irregularities in the Local Magnetic Field}

The scale of irregularities in the magnetic field is of considerable interest, particularly in the studies of cosmic rays. Fermi (1949) initially proposed the existence of a random field component to explain the isotropy of cosmic rays. More recently, Jokipii and Parker (1969) and others have argued that such a component would enable the escape of cosmic rays from the Galaxy in the relatively short period of time implied from observations of abundances of light cosmic-ray nuclei. Using observations of $20-\mathrm{cm}$ polarization in extragalactic sources, Bologna et al. (1965) argued that a decrease in 
TABLE I

Values of longitudinal magnetic fields derived from pulsar observations (Manchester, 1973)

\begin{tabular}{|c|c|c|c|}
\hline PSR & $l$ & $b$ & $\begin{array}{l}\bar{B}_{\mathrm{L}}^{\mathrm{a}} \\
(\mu \mathrm{G})\end{array}$ \\
\hline $0031-07$ & $110^{\circ}$ & $-70^{\circ}$ & $+1.1 \pm 0.1$ \\
\hline $0105+65$ & 125 & +3 & $-1.2 \pm 0.2$ \\
\hline $0138+59$ & 129 & -2 & $-1.8 \pm 0.2$ \\
\hline $0301+19$ & 161 & -33 & $-0.65 \pm 0.02$ \\
\hline $0329+54$ & 145 & -1 & $-2.93 \pm 0.02$ \\
\hline $0450-18$ & 217 & -34 & $+0.46 \pm 0.06$ \\
\hline $0525+21$ & 184 & -7 & $-0.960 \pm 0.006$ \\
\hline $0531+21$ & 185 & -6 & $-0.92 \pm 0.02$ \\
\hline $0611+22$ & 188 & +2 & $+0.88 \pm 0.02$ \\
\hline $0628-28$ & 237 & -17 & $+1.58 \pm 0.02$ \\
\hline $0740-28$ & 244 & -2 & $+2.50 \pm 0.01$ \\
\hline $0809+74$ & 140 & +32 & $-2.5 \pm 0.3$ \\
\hline $0818-13$ & 236 & +13 & $-0.08 \pm 0.05$ \\
\hline $0823+26$ & 197 & +32 & $+0.37 \pm 0.02$ \\
\hline $0833-45$ & 264 & -3 & $+0.59^{\mathrm{b}}$ \\
\hline $0834+06$ & 220 & +26 & $+2.3 \pm 0.3$ \\
\hline $0950+08$ & 229 & +44 & $+0.7 \pm 0.3$ \\
\hline $1112+50$ & 155 & +61 & $+0.43 \pm 0.07$ \\
\hline $1133+16$ & 242 & +69 & $+0.99 \pm 0.06$ \\
\hline $1237+25$ & 252 & +87 & $-0.07 \pm 0.05$ \\
\hline $1508+55$ & 91 & +52 & $+0.05 \pm 0.04$ \\
\hline $1604-00$ & 11 & +35 & $+0.75 \pm 0.12$ \\
\hline $1642-03$ & 14 & +26 & $+0.58 \pm 0.09$ \\
\hline $1706-16$ & 6 & +14 & $-0.12 \pm 0.05$ \\
\hline $1749-28$ & 1 & -1 & $+2.30 \pm 0.05$ \\
\hline $1818-04$ & 26 & +5 & $+1.0 \pm 0.1$ \\
\hline $1822-09$ & 24 & +1 & $+4.4 \pm 0.2$ \\
\hline $1919+21$ & 56 & +3 & $-1.80 \pm 0.06$ \\
\hline $1929+10$ & 47 & -4 & $-3.3 \pm 0.7$ \\
\hline $1933+16$ & 52 & -2 & $-0.015 \pm 0.003$ \\
\hline $2016+28$ & 68 & -4 & $-3.0 \pm 0.2$ \\
\hline $2020+28$ & 69 & -5 & $-3.74 \pm 0.02$ \\
\hline $2021+51$ & 88 & +8 & $-0.36 \pm 0.05$ \\
\hline $2045-16$ & 31 & -33 & $-1.15 \pm 0.04$ \\
\hline $2111+46$ & 89 & -1 & $-1.95 \pm 0.03$ \\
\hline $2154+40$ & 90 & -11 & $-0.76 \pm 0.04$ \\
\hline $2217+47$ & 98 & -8 & $-1.00 \pm 0.05$ \\
\hline $2319+60$ & 112 & -1 & $-2.8 \pm 0.3$ \\
\hline
\end{tabular}

${ }^{a}$ A positive field component is directed towards the observer.

${ }^{b}$ Komesaroff et al. (1971).

the mean polarization with decreasing latitude indicated an increase in depolarization with increasing line of sight through the Galaxy and attributed this depolarization to irregularities on a scale of about $1 \mathrm{pc}$. Their conclusions were rejected by Gardner et al. (1969) who determined that the mean depolarization between $20 \mathrm{~cm}$ and 11 $\mathrm{cm}$ was essentially independent of latitude. However, despite the smooth variation 
of Faraday rotation over ten square degrees of Cen A (Cooper et al., 1965), an extended source at latitude $b=20^{\circ}$, the rapid variation in rotation measure from source to source in some galactic directions (see Figure 2) and across some supernova remnants (Kundu and Velusamy, 1972) is evidence of considerable irregularities in the product $n_{\mathrm{e}} B_{L}$. Any variation in sign is an indication that $B_{L}$ is varying; otherwise the changes

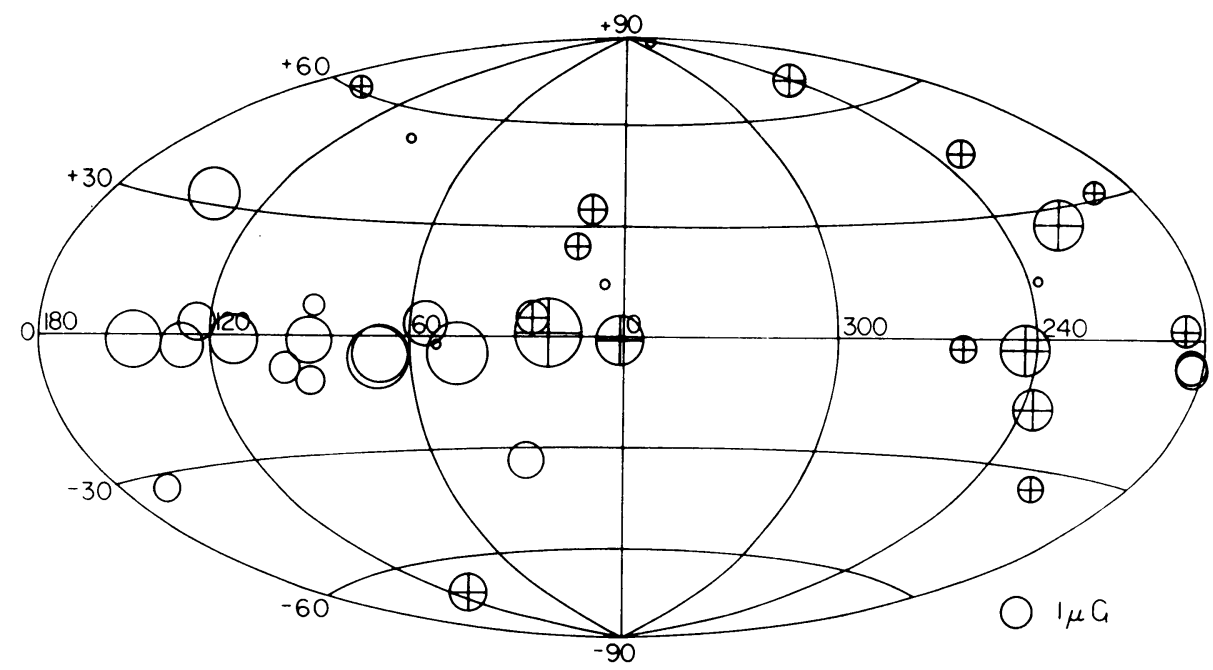

Fig. 3. The distribution with galactic coordinates of the line-of-sight components of magnetic field that were derived from observations of pulsars (Manchester, 1973). The diameters of the circles are proportional to the magnitudes of the derived field; circles containing a positive sign represent a field directed towards the observer.

could be due to a variation of $n_{\mathrm{e}}$ alone. From a statistical analysis of the rotation measures of extragalactic sources, Jokipii and Lerche (1969) deduced that the product $n_{\mathrm{e}} B$ was constant only over distances of $250 \mathrm{pc}$. With more extensive data, Wright (1973) derived a scale of 100-200 pc. However, as mentioned earlier, the variations in rotation measure may not be due only to the local magnetic field, and therefore the scale may be larger than calculated.

In an investigation of the fluctuations of the magnetic field strength, Wright (1973) concluded that the variations could be as high as two to three times the average value. Since the rotation measures of extragalactic sources were used in the analysis, the values may be too high. From observations of pulsars, Manchester (1973) deduced that the strength of the local field fluctuates by only $50 \%$ of the average value. The pulsar sample is small, and not well-distributed with galactic coordinates, and the best estimate probably lies somewhere between the two values.

(b) THE POLARIZATION OF THE GALACTIC BACKGROUND RADIATION

The polarization of the synchrotron radio emission from the galactic background 
yields information about the field transverse to the line of sight, in contrast to Faraday rotation which depends on the line-of-sight component. As a tracer of the local magnetic field, it has considerable value, since the substantial polarization observed between the frequencies $400-1400 \mathrm{MHz}$ is believed to have an origin limited by Faraday depolarization to distances within a few hundred parsec of the Sun. In one of the first investigations, Berkhuijsen et al. (1964) interpreted the directions of polarization and the Faraday rotation near $l=140^{\circ}$ in terms of a field parallel to the galactic plane and directed towards $l=50^{\circ}$. At about the same time Mathewson and Milne (1965) and Mathewson et al. (1966) deduced a similar result (but with a direction $l=70^{\circ}$ ) from the location of the great circle containing the most polarized emission, and from the intrinsic directions of polarization of this emission. A recent, more detailed study by Berkhuijsen (1971) added further support to these conclusions. She showed that the percentage polarization at $829 \mathrm{MHz}$ varied with galactic latitude and longitude in a manner consistent with a field aligned parallel to the galactic plane and directed towards $l=60^{\circ}$.

The first attempts at interpreting the polarization results in terms of small-scale structure of the magnetic field were made by Bingham and Shakeshaft (1967) and Bingham (1967). The most detailed results, although limited to directions containing the spurs of radio emission, were obtained by Spoelstra (1972), who used observations at several frequencies to derive the intrinsic position angles of polarization. The spurs form parts of the loops of radiation that are shown schematically in Figure 2, and which have been fully discussed in articles by Haslam et al. (1971), and Berkhuijsen et al. (1971). For the North Polar Spur, the region of Loop I which extends north of the plane at longitude $l=30^{\circ}$, Spoelstra's results at $1415 \mathrm{MHz}$ for latitudes north of $+50^{\circ}$ are shown in Figure 4. Since the Faraday rotation in these directions is small, the directions of polarization are consistent with a magnetic field component extending along the ridge of radio emission. If the distance of the loop is $50-100 \mathrm{pc}$, and the electron density in the intervening space is $0.06 \mathrm{~cm}^{-3}$, the low rotation measure of $4 \mathrm{rad} \mathrm{m}^{-2}$ yields a mean longitudinal field strength of $1-2 \mu \mathrm{G}$. At low latitudes, the field in the direction of the spur is aligned parallel to the galactic equator, and may represent the field associated with the radio emission in the plane of the Galaxy. Similar field configurations were also obtained for the Cetus Arc, a spur of radio emission defining loop II near $l=150^{\circ}$, and for loop III. For the former region, the rotation measures are lower than for background extragalactic sources, which is evidence that the spur is a local feature.

Thus it appears that a detailed study of the polarization of the galactic synchrotron radiation may provide the best means of investigating the fine structure of the local magnetic field. The results are reasonably consistent with those obtained from the distribution of Faraday rotation - the discrepancy in the direction of the large-scale field may indicate that within a few hundred parsec of the Sun the field orientation differs from that at larger distances. On the other hand, the spatial distribution of the thermal electrons responsible for the Faraday rotation may not be the same as that of the relativistic electrons producing the synchrotron radiation. 


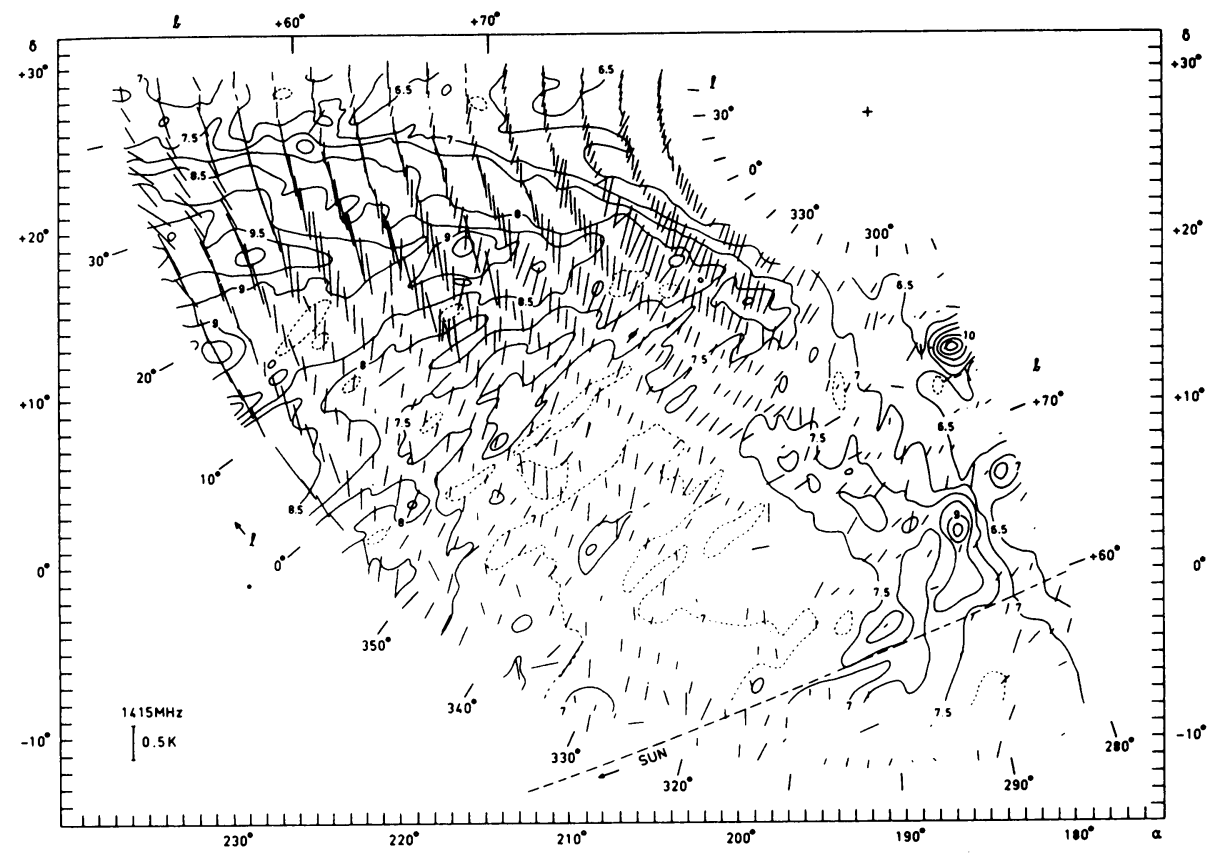

Fig. 4. Polarization measurements at $1415 \mathrm{MHz}$ for the top of the North Polar Spur (Figure 4, Spoelstra, 1972). The vectors show the polarization beam brightness temperatures and directions of polarization; the curves are contours of total beam brightness temperature.

\section{(c) THE ZEEMAN EFFECT}

The Zeeman splitting of the circularly polarized components of spectral lines is proportional to the strength of the line-of-sight magnetic field in an emitting or absorbing region. To date the results of investigations have been disappointing because the uncertainties in the measurements have exceeded a few microgauss. The absorption-line studies of HI by Davies et al. (1968), Brooks et al. (1971), and Verschuur (a series of observations best summarized by Verschuur (1971)), have yielded only the few positive results listed in Table II. The values are considerably higher than for the

TABLE II

Estimated longitudinal magnetic fields from H I Zeeman splitting

\begin{tabular}{lcc}
\hline $\begin{array}{l}\text { Continuum } \\
\text { source }\end{array}$ & $\begin{array}{l}\text { Line } \\
\text { velocity } \\
\left(\mathrm{km} \mathrm{s}^{-1}\right)\end{array}$ & $\begin{array}{l}\text { Observed } \\
\text { field }^{\mathbf{a}} \\
(\mu \mathrm{G})\end{array}$ \\
\hline Tau A & +11 & -6 \\
Ori A & +7 & -50 \\
M17 & +14 & +30 \\
Cas A & -38 & +18 \\
& -48 & +11
\end{tabular}

a A negative field is towards observer. 
fields observed by other techniques in other parts of the Galaxy, and probably represent compressed magnetic fields associated with high-density gas in, or in the immediate vicinity of, each source (Verschuur, 1970). The technique will be of little interest for the investigation of the general galactic field until it is possible to detect field strengths as low as $1 \mu \mathrm{G}$.

A similar conclusion applies to the investigations carried out at $\mathrm{OH}$ frequencies. The observations of Zeeman splitting in $\mathrm{OH}$ absorption (Turner and Verschuur, 1970) yielded upper limits in the range 30-130 $\mu \mathrm{G}$. On the other hand, the presence of features which are similar in intensity, but displaced in velocity, in emission-line profiles measured with right and left circular polarization, has been interpreted in terms of fields with strengths of a few milligauss in high-density regions of W3 (Davies et al., 1966; Yen et al., 1969), W49 (Davies et al., 1966) W75 and W3OH (Rydbeck et al., 1970), and NGC 6334 (Gardner et al., 1970).

\section{(d) OTHER PHENOMENA CORRELATED WITH MAGNETIC FIELD DIRECTIONS}

\section{(i) Interstellar Clouds}

Interstellar clouds can be elongated under the action of magnetic fields (Mansfield, 1973). The existence of dust clouds aligned parallel to magnetic fields has been noted in the Galaxy (Shain, 1955) and in other galaxies (Appenzeller, 1967), and Verschuur (1970) has presented evidence that $\mathrm{HI}$ clouds are similarly elongated. In addition, Berkhuijsen et al. (1971) have pointed out that neutral hydrogen features near the loops of radio emission are extended parallel to the loops. However, the subject has not been investigated in detail; nor has the possibility that the elongation might be due to causes not related to magnetic fields.

\section{(ii) Supernova Remnants}

According to the theoretical models developed for supernova remnants by van der Laan (1962), the remnant has an observed structure that is related to the magnetic field transverse to the line of sight. The structure of two remnants at a relatively high galactic latitude (Whiteoak and Gardner, 1968) and a statistical study by Shaver (1969) supported the models. However, a more extensive study by Milne (1970) failed to yield any correlation between structure and the orientation of the galactic plane. Therefore, the structure of remnants may not be of much use as field tracers, although it is worth noting that Spoelstra (1973) interpreted the galactic loops as supernova remnants, and suggested that the structure of these features is consistent with a magnetic field directed towards $l=40^{\circ}$, a longitude not much different from the values quoted earlier in this review.

\section{Results at Optical Wavelengths}

According to the generally accepted model of Davis and Greenstein (1951), starlight is polarized by foreground dust particles aligned along a magnetic field, with a plane 
of polarization parallel to the field direction. The original model required a relatively high field strength, but a modified version (Jones and Spitzer, 1967) is effective with a more realistic value of $3 \mu \mathrm{G}$. Means of alignment other than by the paramagnetic absorption of the Davis-Greenstein mechanism have been sought - e.g., Martin (1971) has suggested that the field influences the alignment of the particles by causing a precession of the angular momentum of charged grains. Other investigators (e.g. Harwit, 1970; Michel and Yahil, 1973) have questioned on theoretical grounds whether the optical polarization shows unambigously the magnetic field orientation. However, the use of the direction of polarization as a tracer of magnetic fields seems to be supported by studies of the Magellanic Clouds (Mathewson and Ford, 1970b) and other galaxies (Appenzeller, 1968) where, in general, the direction of polarization and galactic structure are correlated. The correlation fails, as expected, in regions of reflected radiation; it also fails in lanes of obscuration that cross the spiral arms, in which case the directions of polarization are parallel to the lanes and presumably delineate the field associated with the dust.

For the Galaxy, the first extensive study, involving distant early-type stars (Hiltner, 1956), suggested a magnetic field aligned along the local spiral arm. Observations of more than 1600 southern stars by Klare et al.(1971) indicated that the field was directed towards $l=80^{\circ}$. However, in the most extensive investigation, of 7000 stars including many nearby objects, Mathewson (1968) noted that the directions of polarization of stars within $500 \mathrm{pc}$ of the Sun were not consistent with a magnetic field parallel to the galactic plane. He interpreted the distribution of these directions in latitude and longitude in terms of a tightly-wound helical field with an axis in the directions $l=90^{\circ}, 270^{\circ}$.

The elegant concept of a local helical field, although in existence in one form or another for some years (Ireland, 1961), has not gained wide acceptance, even though Mathewson (1968) has claimed that it accounts for not only the polarization, but also the distribution of the spurs of galactic radio emission. For instance, an analysis by Seymour (1969), based on a smaller number of stars within $500 \mathrm{pc}$ of the Sun, yielded a field parallel to the galactic plane and directed towards $l=50^{\circ}$, with irregularities on the scale of about $30 \mathrm{pc}$. In addition, the results showed a prominent field extension previously noted by Bingham (1967) along the North Polar Spur. The scale of the irregularities was less than the value $(150 \mathrm{pc})$ derived by Jopikii et al. (1969) using a more distant sample of stars. In an independent investigation, Appenzeller (1968) claimed that the polarization vectors for stars near the south galactic pole were inconsistent with a helical field.

Because of this difference in interpretation, it is of interest to examine the basic observations. Figure 5 shows the distribution of polarization vectors (Mathewson and Ford, 1970a) that was analysed by Mathewson(1968). The vectors near the galactic equator are consistent with a major component of a magnetic field parallel to the plane and directed towards $l=50^{\circ}-80^{\circ}$, at which longitudes there is a great confusion in vector direction consistent with a purely longitudinal field. In a recent review of these observations. Verschuur (1972) concluded that the most likely direction was 
$l=50^{\circ}$, arguing that the directions near $l=80^{\circ}$ contained the Cygnus $\mathrm{X}$ complex which could contribute to the scatter in the polarization vectors. He also argued that directions near $l=80^{\circ}$ were not significant from the viewpoint of spiral structure, but there is a prominent spiral feature at $l=80^{\circ}$ in the distribution of neutral hydrogen (see review by Kerr (1969)). At higher galactic latitudes, the dominating feature is the

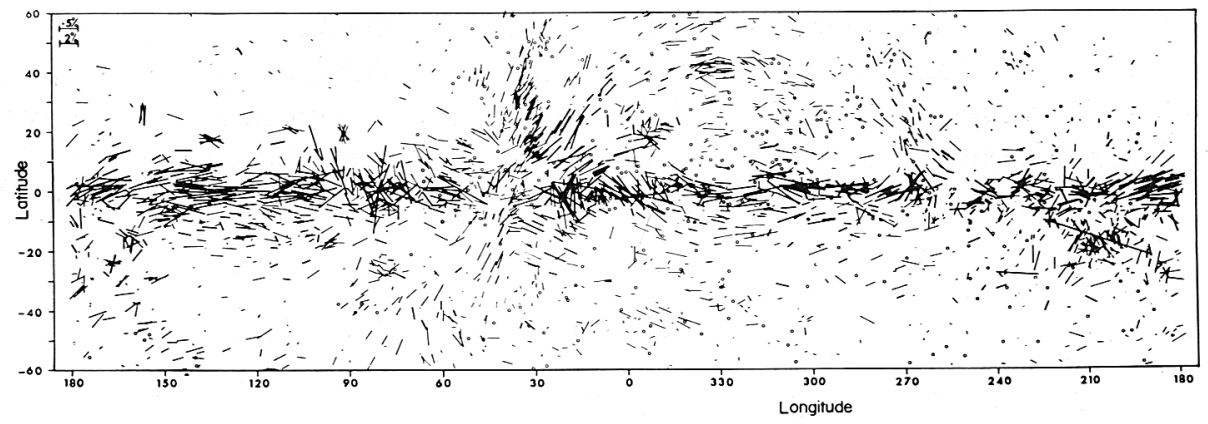

Fig. 5. The distribution with galactic coordinates of the vectors of polarization of starlight (Mathewson and Ford, 1970a). The length of each vector is proportional to the percentage polarization $P$. Small circles are drawn about stars with $P<0.08 \%$.

magnetic loop that is probably associated with the North Polar Spur. The loop is local, since it can be traced in the distribution of vectors for stars in the distance range 50-100 pc (Figure 3a, Mathewson and Ford, 1970a). Another loop can be traced between the longitudes $l=45^{\circ}$ and $l=160^{\circ}$, the direction of the Cetus Arc. The conflict in interpretation depends largely on whether the loops are regarded as parts of an overall helical configuration or whether they are independent irregularities in the local field structure.

Although the observations can be qualitatively interpreted in terms of field structure that is also deduced from studies of radio emission, the interpretation of the optical results in terms of a local helical field is not convincingly disproved. However, a helical field is not consistent with the distribution of Faraday rotation for extragalactic sources (Wright, 1973) or pulsars (Manchester, 1973), or the distribution of galactic radio emission (Spoelstra, 1972). On the basis of the obvious irregularities that exist in the spiral structure of most galaxies, a large-scale field extending along a spiral arm, plus small-scale loops associated with spurs of radio emission, lanes of dust, or spiral arm extensions, seems the more realistic interpretation.

\section{Conclusion}

The main points of this review are:

(i) Observations at radio and optical wavelengths are consistent with a large-scale magnetic field parallel to the galactic equator, which is directed towards longitude $l=50^{\circ}-90^{\circ}$ and which has a field strength of about $2 \mu \mathrm{G}$. Within a few hundred parsecs 
of the Sun, the direction may be $l=50^{\circ}$, while at larger distances, the direction derived from the observations of Faraday rotation $\left(l=90^{\circ}\right)$ appears more appropriate. On the other hand, the variation in direction could arise because the interstellar dust producing the optical polarization is differently distributed with respect to the synchrotron emission and the magnetoionic plasma.

(ii) Within a few hundred parsecs of the Sun, the field contains several loops associated with regions containing spurs of radio emission and interstellar dust.

(iii) Statistical studies suggest that there are field irregularities with a scale of about 100-200 pc. Within these regions, the field strength may be as much as twice the average value. In addition, compressed fields with strengths as high as several milligauss have been detected in several high-density regions in the Galaxy.

\section{References}

Appenzeller, I.: 1967, Publ. Astron. Soc. Pacific 79, 600.

Appenzeller, I.: 1968, Astrophys. J. 151, 907.

Berge, G. L. and Seielstad, G. A. : 1967, Astrophys. J. 148, 367.

Berkhuijsen, E. M.: 1971, Astron. Astrophys. 14, 359.

Berkhuijsen, E. M., Brouw, W. N., Muller, C. A., and Tinbergen, J. A.: 1964, Bull. Astron. Inst. Neth. $17,465$.

Berkhuijsen, E. M., Haslam, C. G. T., and Salter, C. J.: 1971, Astron. Astrophys. 14, 252.

Bingham, R. G.: 1967, Monthly Notices Roy. Astron. Soc. 137, 157.

Bingham, R. G. and Shakeshaft, J. R.: 1967, Monthly Notices Roy. Astron. Soc. 136, 347.

Bologna, J. M., McClain, E. F., Rose, W. K., and Sloanaker, R. M.: 1965, Astrophys. J. 142, 106.

Brooks, J. W., Murray, J. D., and Radhakrishnan, V.: 1971, Astrophys. Letters 8, 121.

Cooper, B. F., Price, R. M., and Cole, D. J.: 1965, Australian J. Phys. 18, 589.

Davies, R. D.: 1972, Monthly Notices Roy. Astron. Soc. 160, 381.

Davies, R. D., Booth, R. S., and Wilson, A. J.: 1968, Nature 220, 1207.

Davies, R. D., de Jager, D., and Verschuur, G. L. : 1966, Nature 209, 974.

Davis, L. and Greenstein, J. L.: 1951, Astrophys. J. 114, 206.

Fermi, E.: 1949, Phys. Rev. 75, 1169.

Gardner, F. F. and Davies, R. D.: 1966, Australian J. Phys. 19, 129.

Gardner, F. F. and Whiteoak, J. B.: 1963, Nature 197, 1162.

Gardner, F. F., Whiteoak, J. B., and Morris, D.: 1967, Nature 214, 371.

Gardner, F. F., Morris, D., and Whiteoak, J. B.: 1969, Australian J. Phys. 22, 813.

Gardner, F. F., Ribes, J. C., and Goss, W. M.: 1970, Astrophys. Letters 7, 51.

Harwit, M.: 1970, Nature 226, 61.

Haslam, C. G. T., Kahn, F. D., and Meaburn, J.: 1971, Astron. Astrophys. 12, 388.

Hiltner, W. A.: 1956, Astrophys. J. Suppl. 2, 389.

Hollinger, J. P., Mayer, C. H., and Menella, R. A.: 1964, Astrophys. J. 140, 656.

Ireland, J. G.: 1961, Monthly Notices Roy. Astron. Soc. 122, 461.

Jokipii, J. R. and Lerche, I.: 1969, Astrophys. J. 157, 1137.

Jokipii, J. R. and Parker, E. N. : 1969, Astrophys. J. 155, 777.

Jokipii, J. R., Lerche, I., and Schommer, R. A.: 1969, Astrophys. J. Letters 157, L119.

Jones, R. V. and Spitzer, L.: 1967, Astrophys. J. 147, 943.

Kerr, F. J.: 1969, Ann. Rev. Astron. Astrophys. 7, 39.

Klare, G., Neckel, T., and Schnuur, G.: 1971, Astron. Astrophys. 11, 155.

Komesaroff, M. M., Ables, J. G., and Hamilton, P. A.: 1971, Astrophys. Letters 9, 101.

Kundu, M. R. and Velusamy, T.: 1972, Astron. Astrophys. 20, 237.

Lerche, I.: 1970, Astrophys. Space Sci. 6, 481.

Manchester, R. N.: 1972, Astrophys. J. 172, 43.

Manchester, R. N.: 1973, Astrophys. J. 186, 637. 
Mansfield, V. N.: 1973, Astrophys. J. 179, 815.

Martin, P. G.: 1971, Monthly Notices Roy. Astron. Soc. 153, 279.

Mathewson, D. S. : 1968, Astrophys. J. Letters 153, L47.

Mathewson, D. S. and Ford, V. L.: 1970a, Mem. Roy. Astron. Soc. 74, 139.

Mathewson, D. S. and Ford, V. L.: 1970b, Astrophys. J. Letters 160, L43.

Mathewson, D. S. and Milne, D. K.: 1965, Australian J. Phys. 18, 635.

Mathewson, D. S., Broten, N. W., and Cole, D. J.: 1966, Australian J. Phys. 19, 93.

Michel, F. C. and Yahil, A.: 1973, Astrophys. J. 179, 771.

Milne, D. K.: 1970, Australian J. Phys. 23, 425.

Mitton, S.: 1972, Monthly Notices Roy. Astron. Soc. 155, 373.

Mitton, S.: 1973, Astrophys. Letters 13, 19.

Morris, D. and Berge, G. L.: 1964, Astrophys. J. 139, 1388.

Piddington, J. H.: 1972, Cosmic Electrodyn. 3, 129.

Rydbeck, O. E. H., Kollberg, E., and Elldér, J.: 1970, Astrophys. J. Letters 161, L25.

Seymour, P. A. H.: 1969, Monthly Notices Roy. Astron. Soc. 142, 33.

Shain, G. A.: 1955, Astron. Zh. 32, 381.

Shaver, P. A.: 1969, Observatory 89, 227.

Spoelstra, T. A. T.: 1972, Astron. Astrophys. 21, 61.

Spoelstra, T. A. T.: 1973, Astron. Astrophys. 24, 149

Turner, B. E. and Verschuur, G. L.: 1970, Astrophys. J. 162, 341.

van der Laan, H.: 1962, Monthly Notices Roy. Astron. Soc. 124, 125.

Verschuur, G. L.: 1970, in H. J. Habing (ed.), 'Interstellar Gas Dynamics', IAU Symp. 39, 150.

Verschuur, G. L.: 1971, Astrophys. J. 165, 651.

Verschuur, G. L.: 1972, Review Paper, IAU Colloquium 23.

Verschuur, G. L.: 1973a, Astron. Astrophys. 22, 139.

Verschuur, G. L.: 1973b, Astron. Astrophys. 27, 407.

Whiteoak, J. B. and Gardner, F. F.: 1968, Astrophys. J. 154, 807.

Wright, W. E.: 1973, 'Polarization Observations of 3C Radio Sources and Galactic Faraday Rotation',

$\mathrm{Ph} . \mathrm{D}$. Thesis, California Institute of Technology.

Yen, J. L., Zuckerman, B., Palmer, P., and Penfield, H.: 1969, Astrophys. J. Letters 156, L27.

\section{J. B. Whiteoak}

Division of Radiophysics, CSIRO,

P.O. Box 76, Epping, N.S.W. 2121, Australia

\section{DISCUSSION}

MacRae: Based on extensive new data on rotation measures, Kronberg and Vallee have developed a model of the local galactic magnetic field. Data on distances of stars and optical polarization are combined with the $R M$ 's to demonstrate a local irregularity in the vicinity of the Sun, superimposed on a general spiral arm magnetic field. (Nature 246, 49 (1973)).

Whiteoak: There are two interpretations on that. The polarization of starlight and the galactic radio emission suggests that the direction of the field within $15-200 \mathrm{pc}$ of the Sun suggests a direction of $\sim 66^{\circ}$, whereas the extragalactic polarization suggests $\sim 90^{\circ}$. On the other hand, one might interpret this as a difference in the distribution of the nonthermal electrons responsible for synchrotron radiation and galactic radio emission and the dust which is causing the starlight polarization.

Mathewson: I split up the 7000 stars for which optical polarization measurements had been made into the distance intervals $0-50 \mathrm{pc}, 50-100 \mathrm{pc}$ and so on up to about $3 \mathrm{kpc}$. An analysis of the directions of the $E$ vectors in each group enabled me to determine the spatial extent of the helical and longitudinal magnetic fields. (Proc. Astron. Soc. Australia, 1 (1969)). The helical field only occupies a volume within about $300 \mathrm{pc}$ of the Sun whilst the longitudinal field which is in the direction $l=90^{\circ}$ and $270^{\circ}$ is more widespread and is probably the general spiral arm magnetic field. I would like to emphasize that this analysis of the optical polarization data gives a longitudinal field as well as helical because in recent years authors of papers on galactic magnetic fields (as in your present review paper) only seem to refer 
to the helical field. I would also like to point out that only six out of the 38 pulsars investigated by Manchester lie within the helical field region while the other 32 lie in the longitudinal field region as defined by the optical polarization vectors.

To sum up there is good agreement between the model based on optical polarization work and the other methods of determining the structure of the galactic magnetic field.

Van Woerden: Does this mean that both sides of parties agree to the same models?

Whiteoak: Yes.

F. G. Smith: Polarization measurements at longer wavelengths show both depolarization and rotation. Away from the Spur regions there is relatively little rotation, indicating the presence of a random field component with rms value comparable to the organized field. In the Spur region there is more rotation, showing that there is a component of field in the line of sight, between the spur source and the Sun.

Whiteoak: The extragalactic source data at high latitude particularly in the direction of the North Polar Spur gives a rotation measure $\sim 4 \mathrm{rad} \mathrm{m}^{2}$. This is in accordance with what you were saying. This is not a very large rotation compared to what one can get close to the plane. This rotation doesn't affect the alignment of the fields to any great extent; it doesn't affect the interpretation. 